\title{
Immunohistochemical Pattern of MLH1/MSH2 Expression Is Related to Clinical and Pathological Features in Colorectal Adenocarcinomas with Microsatellite Instability
}

Giovanni Lanza, M.D., Roberta Gafà, M.D., Iva Maestri, M.Sc., Alessandra Santini, M.D., Maurizio Matteuzzi, M.Sc., Luigi Cavazzini, M.D.

Department of Experimental and Diagnostic Medicine, Section of Anatomic Pathology, University of Ferrara (GL, RG, IM, MM, LC); and Division of Clinical Oncology, St. Anna Hospital (AS), Ferrara, Italy

Detection of colorectal carcinomas with highfrequency microsatellite instability (MSI-H) is clinically important for several reasons. Recent studies suggested that immunohistochemical analysis of MLH1 and MSH2 expression is a rapid and accurate method for identifying large bowel tumors of the MSI-H phenotype. In this study, we evaluated by immunohistochemistry MLH1 and MSH2 protein expression in 132 MSI-H, 23 MSI-L (low-frequency MSI), and 150 microsatellite stable (MSS) colorectal adenocarcinomas. Loss of MLH1 or MSH2 expression was detected in 120 (90.9\%) MSI-H carcinomas, whereas all MSI-L and MSS tumors showed normal expression of both proteins. Lack of MLH1 nuclear staining was observed much more often than absence of MSH2 nuclear staining (106 and 14 cases, respectively). Among MSI-H carcinomas, MLH1/ MSH2 pattern of expression was significantly related to several clinical and pathological variables. In particular, MSI-H MLH1/MSH2-positive carcinomas were more often located in the distal colon, were more frequently classified as ordinary adenocarcinomas, and were more likely to be well or moderately differentiated, 553 positive, and $<7 \mathrm{~cm}$ in diameter than were MLH1-negative and MSH2negative carcinomas. In addition, MLH1-negative carcinomas were less common among patients with hereditary nonpolyposis colorectal cancer (HNPCC) or suspected HNPCC and in the group of patients aged $<50$ years. Patients with MLH1-negative car-

Copyright (C) 2002 by The United States and Canadian Academy of Pathology, Inc.

VOL. 15, NO. 7, P. 741, 2002 Printed in the U.S.A.

Date of acceptance: April 1, 2002.

This work was supported by grants from MURST and Fondazione Cassa di Risparmio di Ferrara.

Address reprint requests to: Giovanni Lanza, M.D., Dipartimento di Medicina Sperimentale e Diagnostica, Sezione di Anatomia Istologia e Citologia Patologica, Università di Ferrara, Via Fossato di Mortara 64/b, 44100 Ferrara, Italy; e-mail: lng@dns.unife.it; fax: 39-0532-248021.

DOI: 10.1097/01.MP.0000018979.68686.B2 cinomas more frequently died of disease than did patients with MLH1/MSH2-positive and MSH2negative MSI-H tumors, but the difference was not statistically significant. The results of the present investigation strongly indicate that immunohistochemical analysis of MLH1 and MSH2 expression is a practical and reliable method for the routine detection of the vast majority of MSI-H large bowel adenocarcinomas. Our data also point out that MSI-H MLH1/MSH2-positive colorectal carcinomas are characterized by distinctive pathological features.

KEY WORDS: Colorectal carcinoma, DNA mismatch repair, Hereditary nonpolyposis colorectal cancer, Immunohistochemistry, Microsatellite instability, MLH1 gene, MSH2 gene, Pathology, Prognosis.

Mod Pathol 2002;15(7):741-749

High-frequency microsatellite instability (MSI-H) occurs in most colorectal adenocarcinomas from patients with hereditary nonpolyposis colorectal cancer (HNPCC) and in about $15 \%$ of sporadic large bowel cancers. MSI-H tumors develop through a mutational pathway different from that operating in common colorectal adenocarcinomas $(1,2)$ and display distinctive pathological features, such as proximal location, poor differentiation, frequent mucinous or medullary/undifferentiated histotype, and intense peritumoral and intratumoral lymphocytic infiltration (3-5). Besides identifying HNPCC gene carriers (6-8), detection of MSI-H large bowel adenocarcinomas is clinically important for several reasons. Patients with MSI-H cancers demonstrated a better clinical outcome than did patients with common large bowel adenocarcinomas, and recent studies performed on very large series of patients showed that the survival advantage conferred by the MSI-H phenotype is independent of tumor stage 
and other clinical and pathological variables (5, $9-12$ ). In addition, clinical evidence suggests that MSI-H colorectal carcinomas may be particularly sensitive to 5-fluorouracil-based adjuvant chemotherapy $(13,14)$. Finally, patients with MSI-H tumors are thought to be at increased risk of developing metachronous large bowel carcinomas and therefore to require long-term colonoscopic follow-up (15).

MSI-H is determined by defects in the DNA mismatch repair (MMR) system $(16,17)$. In HNPCC, MSI-H is caused by germline mutations of one of the MMR genes, in most cases the MLH1 or MSH2 gene, with somatic inactivation of the remaining wild-type allele (17). In sporadic MSI-H large bowel carcinomas, mutations of MMR genes have been detected at a very low frequency, and MLH1 gene inactivation by promoter hypermethylation is the major mechanism leading to the mutator phenotype (18-20). Genetic or epigenetic inactivation of MLH1 and MSH2 genes is generally associated with loss of immunohistochemical expression of the corresponding protein $(18,20,21)$. Recent studies suggest that immunohistochemical analysis of MLH1 and MSH2 gene product expression may allow one to specifically identify MSI-H colorectal adenocarcinomas $(15,22-26)$. Therefore, immunohistochemistry for MLH1 and MSH2 seems to represent a rapid and accurate methō for the routine detection of these tumors.

In the present investigation, we evaluated MLH1 and MSH2 expression in a large number of MSI-H, MSI-L (low-frequency MSI), and microsatellite stable (MSS) colorectal adenocarcinomas. The relationship between the immunohistochemical pattern of MLH1/MSH2 expression and the clinical and pathological features of MSI-H tumors was also analyzed.

\section{MATERIALS AND METHODS}

The study included 724 colorectal adenocarcinomas surgically resected in the years 1988-1994 and 1999-2000. To obtain a very large number of MSI-H tumors, a nearly equal number of carcinomas of the proximal (caecum, ascending, and transverse colon; $n=338$ ) and distal colon (left colon and rectum; $n=386$ ) were examined. Availability of frozen tissue samples suitable for genetic analyses was the only criterion for selection. Patients with familial adenomatous polyposis or inflammatory bowel disease were excluded.

Tumors were staged according to the TNM staging system (27). Tumor type and grade of differentiation were determined by the criteria of the World Health Organization (28). Carcinomas with a predominant solid growth pattern $(\geq 70 \%$ of the tumor area) and mild or moderate nuclear pleomorphism were classified as medullary adenocarcinomas (29). Pattern of growth, lymphocytic infiltration at the advancing tumor margin, and extramural vein invasion were evaluated according to the criteria of Jass et al. (30). Peritumoral Crohn's-like lymphoid reaction was assessed according to Graham and Appelman (31) and classified as conspicuous (intense) and inconspicuous $(5,29)$.

Detailed clinical follow-up data were available for all patients operated on before December 1994. Personal and family histories of colorectal carcinoma and other cancers were obtained by chart review and in most cases by interview of patients and/or family members. Whenever possible, pathological review of HNPCC-related tumors of all family members was performed. However, for the large majority of patients, only data concerning first-degree relatives were accessible, and no attempt to construct expanded family trees was done.

\section{Microsatellite Analysis}

Representative samples of tumor and corresponding normal mucosa were obtained from fresh surgical specimens, frozen in liquid nitrogen, and stored at $-80^{\circ} \mathrm{C}$. DNA was extracted by a standard phenol-chloroform procedure. Before DNA extraction, from each tumor sample, a frozen section was cut and stained with hematoxylin and eosin to verify by microscopic examination the presence of adequate neoplastic material $(\geq 60-70 \%$ of tumor cells).

In the majority of cases $(n=614)$, MSI was evaluated at six microsatellite loci (D18S58, D18S61, D17S855, D17S786, BAT26, and BAT40) using a fluorescence-based polymerase chain reaction (PCR) method, as reported elsewhere (5, 29). Analysis of the PCR products was performed with an automated laser-activated fluorescent DNA sequencer (ALF; Pharmacia Biotech, Cologno Monzese, Italy) using the Fragment Manager (FM1.1) software (Pharmacia Biotech). More recently, in the last 110 tumors, MSI was analyzed with the Bethesda panel of five microsatellite markers (BAT26, BAT25, D2S123, D5S346, D17S250) (32) and a fluorescence-based multiplex PCR method, using the HNPCC Microsatellite Instability Test kit (Boehringer Mannheim, Mannheim, Germany) according to manufacturer's instructions. PCR products were run in an ABI PRISM 377 DNA sequencer (Perkin-Elmer Applied Biosystems Division, Foster City, CA) and analyzed by the GeneScan 3.1 software (PerkinElmer) (Fig. 1). 


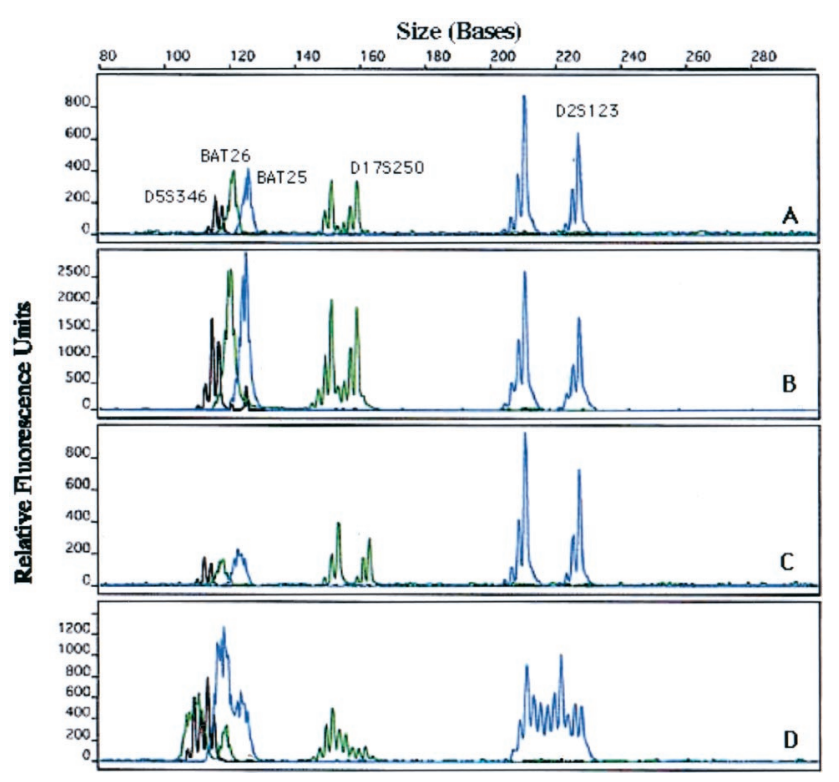

FIGURE 1. Analysis of microsatellite instability in colorectal adenocarcinomas using the five markers of the International Workshop of Bethesda (32) and fluorescence-based multiplex polymerase chain reaction. Fragment pattern of a high-frequency microsatellite instability tumor showing instability at all five loci examined (Lane D: tumor tissue; Lane C: corresponding normal mucosa) and of a microsatellite stable (MSS) carcinoma (Lane B: tumor tissue; Lane A: normal mucosa).

MSI was defined as the presence in the tumor DNA of PCR products of abnormal size compared with the corresponding normal tissue. Tumors demonstrating instability at one or two loci with one set of microsatellite markers were further analyzed with the other microsatellite markers (total number, 10), According to the guidelines of the International Workshop of Bethesda (32), tumors showing instability at $\geq 30 \%$ of microsatellite loci were classified as MSI-H, tumors demonstrating instability at $<30 \%$ of microsatellite loci were classified as MSI-L, and tumors without detectable MSI as MSS (microsatellite stable).

\section{Immunohistochemical Analysis}

Immunohistochemical analysis of MLH1 and MSH2 expression was performed in all MSI-H and MSI-L tumors, and in a consecutive series of 150 unselected MSS carcinomas, following an analytical procedure previously described (5). Briefly, one block of formalin-fixed paraffin-embedded tumor tissue, usually comprising adjacent normal mucosa, was selected per case. Five-micrometer-thick sections were deparaffinized and rehydrated using xylene and alcohol. Before immunostaining, heat-induced antigen retrieval was performed by treatment in a microwave oven (30 min at $600 \mathrm{~W}$ ) of tissue sections immersed in $10 \mathrm{~mm}$ citrate buffer, $\mathrm{pH}$ 6.0. Sections were then incubated overnight at $4^{\circ} \mathrm{C}$ with mouse monoclonal antibodies against MLH1 protein (clone G168-728; PharMingen, San Diego, CA) and MSH2 protein (clone FE11; Oncogene Research Products, Cambridge, MA), both diluted 1:100. An UltraVision streptavidin-biotin peroxidase detection kit (TP060-HL; Lab Vision Corporation, Fremont, CA) was employed as the secondary detection system, and the peroxidase reaction was developed using diaminobenzidine tetrachloride as chromogen. Finally, slides were lightly counterstained with Mayer hematoxylin.

Tumors showing loss of nuclear MLH1 or MSH2 expression were classified as MLH1 or MSH2 negative. Nuclear immunostaining of normal epithelial cells, lymphocytes, and stromal cells served as internal positive controls (Fig. 2). All cases were evaluated independently by two pathologists (GL and RG) without knowledge of clinicopathologic data and MSI status.

Immunohistochemical analysis of p53 protein expression was performed on formalin-fixed paraffinembedded tissue sections using the mouse monoclonal antibody DO-7 (DBS, Pleasanton, CA), as reported in previous studies $(5,29)$. Tumors showing a proportion of stained nuclei of $>10 \%$ were classified as p53 positive.

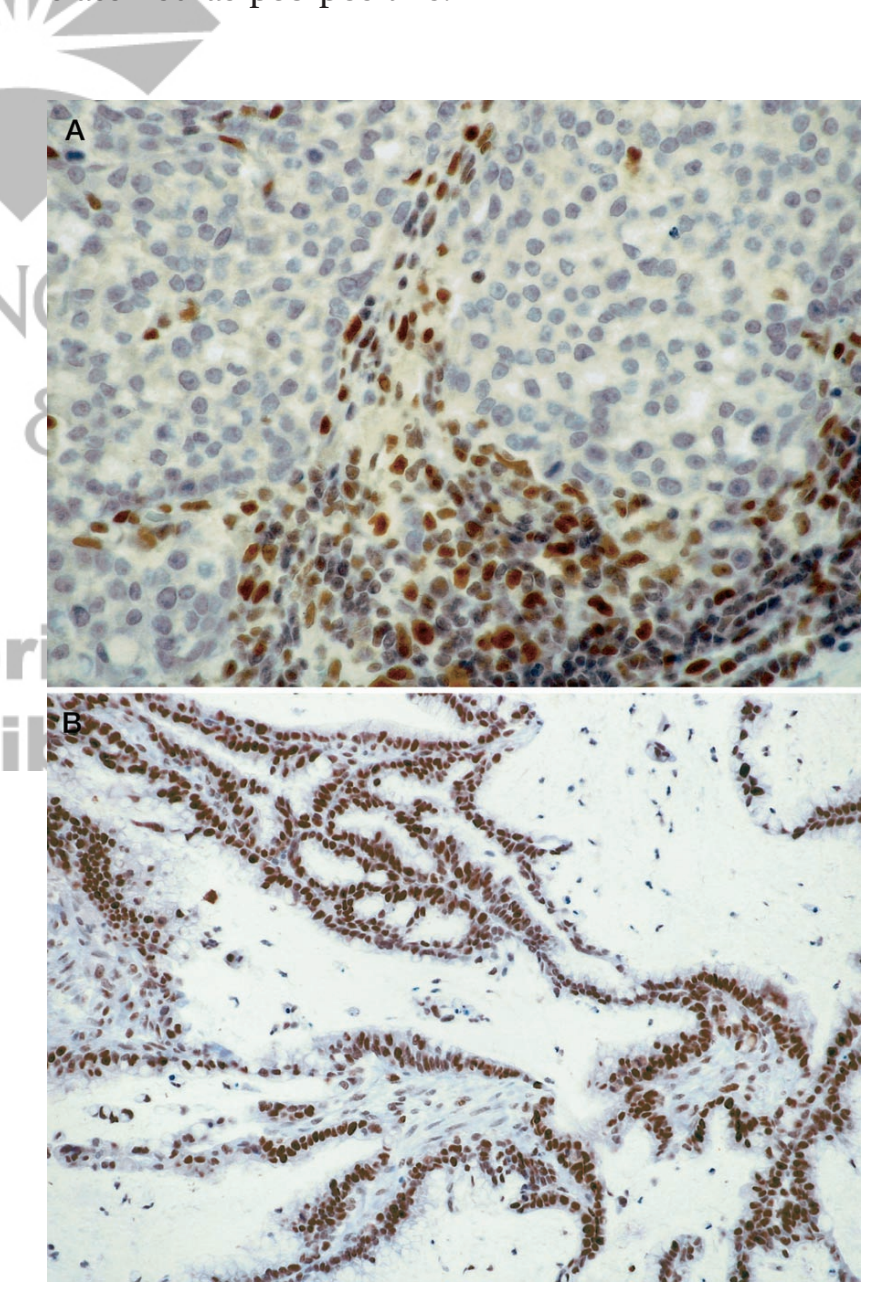

FIGURE 2. A, high-frequency microsatellite instability (MSI-H) medullary adenocarcinoma with complete loss of MSH2 expression. Nuclear staining of lymphocytes in the stroma served as internal positive control. B, mucinous adenocarcinoma with normal MSH2 expression. 
Statistical Analysis

Differences in distributions between the variables examined were assessed with the $\chi^{2}$ test or the Fisher's exact test, as appropriate. Survival curves were generated using the method of Kaplan and Meier and compared by the log rank test. Data from patients who died of causes unrelated to colorectal cancer were censored at the time of death. Probability values of $<.05$ were considered significant. All data were analyzed using SPSS statistical software, Version 8.0 (SPSS Inc., Chicago).

\section{RESULTS}

\section{MSI Status}

Of the 724 colorectal adenocarcinomas examined, $133(18.4 \%)$ were classified as MSI-H, 23 $(3.2 \%)$ as MSI-L, and $568(78.4 \%)$ as MSS. MSI-H tumors occurred much more often in the proximal than in the distal colon $(115 / 338,34.0 \%$ versus $18 /$ $386,4.7 \% ; P<.0001)$. The large majority of MSI-H carcinomas (115 of $133,86.5 \%)$ were sited in the proximal colon, whereas MSI-L tumors were equally distributed in the proximal and distal colon (12 and 11, respectively).

\section{MLH1/MSH2 Expression in MSI-H, MSI-L, and MSS Carcinomas}

Loss of MLH1 or MSH2 expression was detected in $120(90.9 \%)$ of the $132 \mathrm{MSI}-\mathrm{H}$ carcinomas examined (one MSI-H tumor was excluded from/the study because the quality of immunostaining was unsatisfactory). In detail, 106 (80.3\%) MSI-H carcinomas showed complete loss of MLH1 expression and normal immunoreactivity for MSH2 (MLH negative), 14 (10.6\%) displayed complete loss of MSH2 expression and normal immunoreactivity for MLH1 (MSH2 negative), and 12 MSI-H carcinomas (9.1\%) demonstrated normal expression of both MLH1 and MSH2 gene products (MLH1/MSH2 positive; Table 1). There were no MSI-H tumors showing lack of both MLH1 and MSH2 expression. In contrast, nuclear immunoreactivity for MLH1 and MSH2

TABLE 1. Immunohistochemical Pattern of MLH1/MSH2 Expression in MSI-H, MSI-L, and MSS Colorectal Carcinomas

\begin{tabular}{lrccc}
\hline \multirow{2}{*}{ MSI Status } & $n$ & \multicolumn{3}{c}{ Pattern of Immunoreactivity, $n$ (\%) } \\
\cline { 3 - 5 } & & MLH1 & MSH2 & MLH1/MSH2 \\
& & Negative & Negative & Positive \\
\hline MSI-H & 132 & $106(80.3)$ & $14(10.6)$ & $12(9.1)$ \\
MSI-L & 23 & & & $23(100.0)$ \\
MSS & 150 & & & $150(100.0)$ \\
\hline
\end{tabular}

MSI-H, high-frequency microsatellite instability; MSI-L, low-frequency microsatellite instability; MSS, microsatellite stable. proteins was observed in all 150 MSS and 23 MSI-L tumors analyzed (Table 1).

Five patients in the study had two synchronous or metachronous MSI-H carcinomas. In all these cases, the tumors resected from a single patient showed the same pattern of immunoreactivity (MLH1 negative in two patients, MSH2 negative in two patients, and MLH1/MSH2 positive in one patient).

Instability at the BAT26 locus was detected in 125 of the $132(94.7 \%)$ MSI-H tumors. Of the seven carcinomas negative for BAT26 instability, four were MLH1 negative and three were MLH1/MSH2 positive, whereas all the MSH2-negative carcinomas showed BAT26 instability. Six of the seven tumors negative for BAT26 instability showed instability at other mononucleotide loci (BAT40 and/or BAT25). One MLH1/MSH2-positive tumor demonstrated instability only at dinucleotide repeats. All the other MSI-H carcinomas included in the study showed instability at both mononucleotide and dinucleotide loci. There were no differences in the degree of MSI between MLH1-negative, MSH2negative and MLH1/MSH2-positive MSI-H adenocarcinomas (data not shown).

\section{Relationship between MLH1/MSH2 Expression and Clinicopathological Variables in MSI-H Carcinomas}

Among MSI-H tumors, immunohistochemical pattern of MLH1/MSH2 expression was demonstrated to be related to several clinical and pathological variables (Table 2).

MLH1-negative carcinomas occurred more often in women (62.5\%) than MSH2-negative (41.7\%) and MLH1/MSH2-positive (45.5\%) tumors, but the difference was not statistically significant. In addition, MLH1-negative carcinomas developed less frequently in patients aging $<50$ years than did MLH1/MSH2positive $(P=\mathrm{NS})$ and MSH2-negative tumors $(P=$ .005). A close relationship was demonstrated between MLH1/MSH2 immunostaining and tumor site. The large majority (93.4\%) of MLH1-negative, but only $64.3 \%$ of MSH2-negative $(P=.0006)$ and $50 \%$ of MLH1/MSH2-positive carcinomas $(P<.001)$, were located proximal to the splenic flexure. Of the 114 proximal MSI-H tumors, 99 (86.8\%) were MLH1 negative, 9 (7.9\%) were MSH2 negative, and 6 (5.3\%) were MLH1/MSH2 positive. Conversely, 7 (38.9\%) of the 18 distal MSI-H carcinomas were MLH1 negative, 5 (27.8\%) were MSH2 negative, and 6 (33.3\%) were MLH1/MSH2 positive. Furthermore, MLH1-negative and MSH2-negative MSI-H cancers were more often $>7 \mathrm{~cm}$ in diameter larger than were MLH1/MSH2positive ones (54.7, 35.7, and $8.3 \%$, respectively; MLH1 negative versus MLH1/MSH2 positive, $P=$ 
.002). No significant relation was detected between tumor stage and MLH1/MSH2 expression (Table 2).

MLH1/MSH2 immunoreactivity was also related to tumor type (Table 2). MSI-H MLH1/MSH2positive tumors were more often classified as ordinary adenocarcinomas (58.3\%) than were MLH1 negative (17.9\%; $P=.001$ ) and $\mathrm{MSH} 2$ negative (14.3\%) carcinomas. MSI-H MLH1/MSH2-positive carcinomas were also more often well or moderately differentiated (75\% of cases) and p53 positive (50\%) than were the other MSI-H tumors, especially those that were MLH1 negative $(P=.005$ and $P=$ .007 , respectively; Table 2). Because of the small numbers of cases included in both groups, all the above-mentioned differences between MSH2negative and MLH1/MSH2-positive MSI-H tumors did not reach statistical significance. MLH1/MSH2 pattern of expression was not associated with other histopathological variables such as lymphocytic infiltration at the advancing tumor margin (Table 2), Crohn's-like lymphoid reaction (Table 2), pattern of growth, and vein invasion (data not shown).

MSI-L and MSS tumors showed very similar clinical and pathological characteristics (data not shown) and therefore were grouped together and indicated as MSI-L/MSS. MSI-L/MSS carcinomas differed from all MSI-H tumors for nearly all the clinicopathological features examined (Table 2).

Seventy-nine patients with MSI-H carcinomas were operated on before December 1994 and were included in survival analysis. Two patients were Stage I, 44 were Stage II, 27 were Stage III, and 6 were Stage IV. The mean follow-up in surviving patients was 77.1 months (median, 79.3 mo; range,

TABLE 2. Relationship between MLH1/MSH2 Immunoreactivity and Clinicopathological Features in MSI-H Colorectal Carcinomas; Clinical and Pathological Characteristics of MSI-L/MSS Tumors Are Also Reported

\begin{tabular}{|c|c|c|c|c|c|c|c|c|}
\hline \multirow[b]{2}{*}{ Feature } & \multicolumn{6}{|c|}{ MSI-H Tumors, $n(\%)$} & \multirow[b]{2}{*}{$P$} & \multirow{2}{*}{$\begin{array}{l}\text { MSI-L/MSS } \\
\text { Tumors (\%) }\end{array}$} \\
\hline & $\begin{array}{c}\text { MSH2 } \\
\text { Negative }\end{array}$ & & $\begin{array}{c}\text { MLH1 } \\
\text { Negative }\end{array}$ & & $\begin{array}{c}\text { MLH1/MSH2 } \\
\text { Positive }\end{array}$ & All & & \\
\hline \multicolumn{9}{|l|}{ Gender } \\
\hline Male & $7(58.3)$ & NS & 39 (37.5) & NS & $6(54.5)$ & $52(40.9)^{a}$ & NS & $86(49.7)$ \\
\hline Female & $5(41.7)$ & & $65(62.5)$ & & $5(45.5)$ & $75(59.1)$ & & $87(50.3)$ \\
\hline \multicolumn{9}{|l|}{ Age $(y)$} \\
\hline$<50$ & $4(33.3)$ & 0.005 & $6(5.8)$ & NS & $2(18.2)$ & $12(9.5)^{a}$ & $<0.0001$ & $10(5.8)$ \\
\hline $50-70$ & $3(25.0)$ & & $44(42.3)$ & & $3(27.3)$ & $50(39.3)$ & & $113(65.3)$ \\
\hline$>70$ & $5(41.7)$ & & $54(51.9)$ & & $6(54.5)$ & $65(51.2)$ & & $50(28.9)$ \\
\hline \multicolumn{9}{|l|}{ Tumor site } \\
\hline Proximal colon & $9(64.3)$ & 0.0006 & $99(93.4)$ & $<0.001$ & $6(50.0)$ & $114(86.4)$ & $<0.0001$ & $53(30.6)$ \\
\hline Distal colon & $5(35.7)$ & & $7(6.6)$ & & $6(50.0)$ & $18(13.6)$ & & $120(69.4)$ \\
\hline \multicolumn{9}{|l|}{ Tumor size (cm) } \\
\hline$<4$ & $2(14.3)$ & $\mathrm{NS}^{b}$ & $4(3.8)$ & $0.002^{b}$ & $3(25.0)$ & $9(6.8)$ & $<0.0001^{b}$ & $21(12.1)$ \\
\hline $4-7$ & $7(50.0)$ & & $44(41.5)$ & & $8(66.7)$ & $59(44.7)$ & & $135(78.1)$ \\
\hline$>7$ & $5(35.7)$ & & $58(54.7)$ & & $1(8.3)$ & $64(48.5)$ & & $17(9.8)$ \\
\hline \multicolumn{9}{|l|}{ Tumor stage (TNM) } \\
\hline I & $2(14.3)$ & NS & $5(4.7)$ & NS & $3(25.0)$ & $10(7.6)$ & 0.004 & $4(2.3)$ \\
\hline II & $9(64.3)$ & & $55(51.9)$ & & $5(41.6)$ & $69(52.2)$ & & $71(41.1)$ \\
\hline III & $3(21.4)$ & & 38 (35.9) & & $2(16.7)$ & $43(32.6)$ & & $68(39.3)$ \\
\hline IV & & & $8(7.5)$ & & $2(16.7)$ & $10(7.6)$ & & $30(17.3)$ \\
\hline \multicolumn{9}{|l|}{ Tumor type } \\
\hline Adenocarcinoma & $2(14.3)$ & $\mathrm{NS}^{c}$ & $19(17.9)$ & $0.001^{c}$ & $7(58.3)$ & $28(21.2)$ & $<0.0001^{c}$ & $129(74.6)$ \\
\hline Adenocarcinoma with mucus $<50 \%$ & $4(28.6)$ & & $29(27.4)$ & & $2(16.7)$ & $35(26.5)$ & & $22(12.7)$ \\
\hline Mucinous adenocarcinoma & $3(21.4)$ & 0 & $24(22.6)$ & - & $2(16.7)$ & $29(22.0)$ & & $22(12.7)$ \\
\hline Medullary adenocarcinoma & $5(35.7)$ & & $34(32.1)$ & & $1(8.3)$ & $40(30.3)$ & & \\
\hline \multicolumn{9}{|l|}{ Grade of differentiation } \\
\hline Well or moderate & $7(50.0)$ & NS & $36(34.0)$ & 0.005 & $9(75.0)$ & $52(39.4)$ & $<0.0001$ & $127(73.4)$ \\
\hline Poor & $7(50.0)$ & & $70(66.0)$ & & $3(25.0)$ & $80(60.6)$ & & $46(26.6)$ \\
\hline \multicolumn{9}{|l|}{ Lymphocytic infiltration at the tumor margin } \\
\hline Little or none & $8(57.1)$ & NS & $73(68.9)$ & NS & $5(41.7)$ & $86(65.2)$ & NS & $117(67.6)$ \\
\hline Marked or moderate & $6(42.9)$ & & $33(31.1)$ & & $7(58.3)$ & $46(34.8)$ & & $56(32.4)$ \\
\hline \multicolumn{9}{|l|}{ Crohn's-like lymphoid reaction } \\
\hline Inconspicuous & $9(64.3)$ & NS & $62(58.5)$ & NS & $7(58.3)$ & $78(59.1)$ & $<0.0001$ & $125(91.9)^{d}$ \\
\hline Conspicuous & $5(35.7)$ & & $44(41.5)$ & & $5(41.7)$ & $54(40.9)$ & & $11(8.1)$ \\
\hline \multicolumn{9}{|l|}{ p53 expression } \\
\hline Negative & $10(71.4)$ & NS & $88(83.0)$ & 0.007 & $6(50.0)$ & $104(78.8)$ & $<0.0001$ & $68(39.3)$ \\
\hline Positive & $4(28.6)$ & & $18(17.0)$ & & $6(50.0)$ & $28(21.2)$ & & $105(60.7)$ \\
\hline \multicolumn{9}{|l|}{ Vital status ${ }^{e}$} \\
\hline Alive or dead without evidence of disease & $8(100)$ & NS & $47(74.6)$ & NS & $7(87.5)$ & $62(78.5)$ & 0.0002 & $93(54.1)$ \\
\hline Dead of disease & & & $16(25.4)$ & & $1(12.5)$ & $17(21.5)$ & & $79(45.9)$ \\
\hline
\end{tabular}




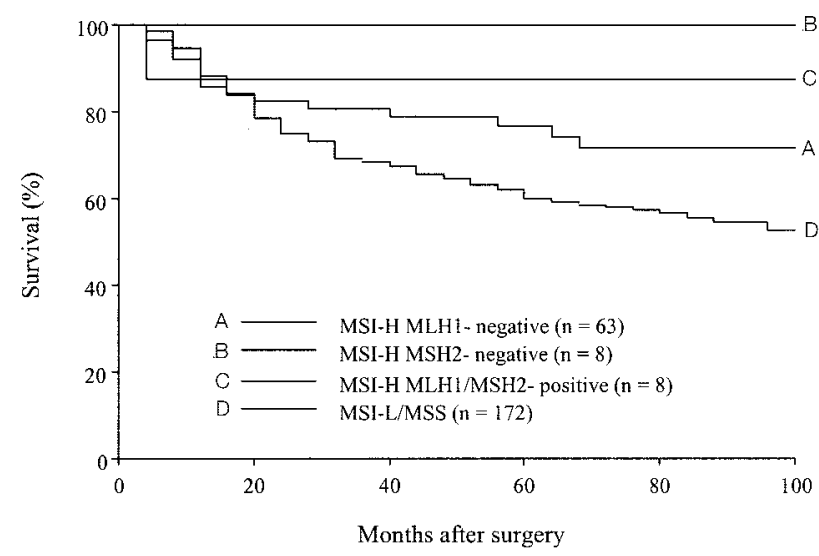

FIGURE 3. Disease-specific survival of colorectal cancer patients by microsatellite instability status and MLH1/MSH2 immunostaining. Among high-frequency microsatellite instability (MSI-H) tumors, patients with MLH1-negative carcinomas more often died of disease than did patients with MLH1/MSH2-positive and MSH2-negative carcinomas, but the differences in survival between the three groups of patients were not statistically significant. Patients with low-frequency microsatellite instability (MSI-L) or microsatellite stable (MSS) tumors showed a less favorable clinical outcome with respect to all patients with MSI-H carcinomas $(P=.0041)$

37 to 114 mo). Among MSI-H tumors, patients with MLH1-negative carcinomas more frequently died of disease (16 of $63,25.4 \%)$ than did patients with MLH1/MSH2-positive (1 of 8, 12.5\%) and MSH2negative carcinomas (none of 8) (Table 2). However, disease-specific survival analysis did not show significant differences between the three groups of patients (Fig. 3). One hundred and seventy-two patients with MSI-L/MSS tumors were available for survival analysis (mean follow-up in surviving patients, 91.6 mo; median, 86.4 mo; range, 61 to 128 mo). Patients with MSI-L/MSS tumors demonstrated a less favorable clinical outcome compared with all patients with MSI-H carcinomas $(P=.0041$; Fig. 3).

Data concerning personal and family histories of cancer could be obtained for 88 (69.3\%) of the 127 patients with MSI-H carcinomas. In the group of patients with HNPCC $(n=3)$ or suspected HNPCC $(n=7), 50 \%$ of the patients had MLH1-negative tumors; 30\%, MSH2-negative tumors; and 20\%, MLH1/MSH2-positive tumors (Table 3). MLH1/ MSH2-positive carcinomas were slightly overrepre- sented (21.4\%) among patients with one firstdegree relative affected by colorectal cancer. In contrast, the large majority of MSI-H tumors arising in patients with a family history negative for colorectal cancer were MLH1 negative (87.5\%) (Table 3). The difference in MLH1/MSH2 expression between the tumors of the three groups of patients was statistically significant $(P=.016)$.

\section{DISCUSSION}

Detection of MSI-H large bowel adenocarcinomas is clinically important for several reasons. It may represent an initial step in the identification of colorectal cancer patients with HNPCC (6-8). Furthermore, patients with MSI-H carcinomas demonstrated in several studies a survival advantage with respect to patients with MSI-L/MSS tumors (5, 9-12). There is also the suggestion that MSI-H carcinomas might be particularly sensitive to 5-fluorouracil-based adjuvant chemotherapy $(13,14)$. Finally, patients with MSI-H tumors are considered to be at increased risk of developing metachronous colorectal cancers and need long-term colonoscopic surveillance (15).

Genetic analysis of MSI status is time consuming and expensive and requires specialized equipment. Therefore, at present it cannot be performed routinely on large bowel adenocarcinomas by histopathology laboratories. Immunohistochemical analysis of MLH1 and MSH2 protein expression represents a rapid, easier, and less costly alternative method for the detection of colorectal tumors of the mutator phenotype, and it could be performed by histopathology laboratories as a routine diagnostic test. In the present investigation, we demonstrated loss of MLH1 or MSH2 expression in a high proportion (90.9\%) of the 132 MSI-H colorectal carcinomas examined. In contrast, nuclear expression of both MLH1 and MSH2 gene products was observed in all the MSS $(n=150)$ and MSI-L $(n=23)$ tumors analyzed. Our data indicate that immunohistochemical analysis of MLH1 and MSH2 expression is a sensitive and specific method for the identification of colorectal tumors of the MSI-H phenotype. Our results are in agreement with previous studies

TABLE 3. Relationship Between MLH1/MSH2 Immunostaining Pattern and Family History of Colorectal Cancer among Patients with MSI-H Large Bowel Carcinomas

\begin{tabular}{|c|c|c|c|c|}
\hline \multirow{2}{*}{$\begin{array}{l}\text { Family History of Colorectal } \\
\text { Cancer }\end{array}$} & \multirow{2}{*}{$\begin{array}{l}\text { No. of Patients } \\
(\%)\end{array}$} & \multicolumn{3}{|c|}{ Pattern of Immunoreactivity (\%) } \\
\hline & & MLH1 Negative & MSH2 Negative & MLH1/MSH2 Positive \\
\hline HNPCC or suspected HNPCC & $10(7.9)$ & $5(50.0)$ & $3(30.0)$ & $2(20.0)$ \\
\hline Positive & $14(11.0)$ & $10(71.4)$ & $1(7.2)$ & $3(21.4)$ \\
\hline Negative & $64(50.4)$ & $56(87.5)$ & $6(9.4)$ & $2(3.1)$ \\
\hline Not available & $39(30.7)$ & $33(84.6)$ & $2(5.1)$ & $4(10.3)$ \\
\hline
\end{tabular}

HNPCC, hereditary nonpolyposis colorectal cancer fulfilling the Amsterdam criteria; suspected HNPCC, one or more first-degree relatives with colorectal or HNPCC-related tumors, and early-onset colorectal cancer or multiple large bowel adenocarcinomas; positive, one first-degree relative affected by colorectal cancer; negative, no first-degree relatives affected by colorectal cancer. 
performed on smaller series of cases, which demonstrated lack of MLH1 or MSH2 immunostaining in a high percentage $(20,22-26,33)$ or even the totality (15) of large bowel adenocarcinomas with a high degree of MSI. Some studies reported lack of MLH1 or MSH2 expression in a small number of non-MSI-H large bowel adenocarcinomas $(25,33)$. In our opinion, and in agreement with findings of other investigators $(25,33)$, this finding was mainly determined by inadequate tissue fixation. We occasionally observed reduced or absent nuclear MLH1 and/or MSH2 staining of tumor cells in areas of otherwise positive MSI-L/MSS carcinomas, but in the same areas, loss of immunoreactivity in the nuclei of nonneoplastic cells was also detected. Nevertheless, it should be pointed out that even if carefully optimized, immunohistochemical stain for MLH1 and MSH2 fails to detect nearly $10 \%$ of MSI-H colorectal carcinomas. In addition, the fraction of HNPCC colorectal cancer patients not associated with MLH1 or MSH2 germline mutations cannot be identified by this method.

In the $132 \mathrm{MSI}-\mathrm{H}$ carcinomas examined, loss of MLH1 expression occurred much more frequently (106 cases, $80.3 \%$ ) than did loss of MSH2 expression (14 cases, 10.6\%). However, among patients younger than 50 years of age and in the group of patients belonging to families with HNPCC or suspected HNPCC, lack of MLH1 and of MSH2 immunostaining were more equally distributed $(50 \%$ and nearly $30 \%$, respectively). Our results are in agreement with those of previous studies, which demonstrated a high prevalence of loss of MLH1 expression in unselected series of MSI-H large bowel carcinomas $(15,23,25,26)$ and nearly equal frequencies of loss of MLH1 and MSH2 staining in series comprising a high number of hereditary cases $(22,24,33)$. Our findings are also in agreement with molecular data indicating that epigenetic inactivation of the MLH1 gene by promoter hypermethylation is the leading cause of mismatch repair deficiency in sporadic MSI-H colorectal adenocarcinomas (18-20), whereas germline mutations in either MLH1 or MSH2 are responsible for HNPCC in roughly equal proportions of cases (17).

MSI-H colorectal carcinomas, both sporadic and HNPCC associated, differ from MSI-L/MSS tumors in several pathological characteristics. MSI-H tumors are predominantly located in the proximal colon and more often display large size, poor differentiation, mucinous or medullary histology, expanding pattern of growth, intense peritumoral Crohn's-like lymphoid reaction, and intratumoral lymphocytic infiltration $(3-5,26,34)$. In addition, the large majority of MSI-H colorectal carcinomas are DNA diploid by flow cytometry and p53 negative by immunohistochemical analysis $(5,33,35)$. The pathological features of the MSI-H carcinomas included in our analysis are consistent with those reported in the majority of previous studies. In fact, $86.4 \%$ of the $132 \mathrm{MSI}-\mathrm{H}$ carcinomas were located in the proximal colon, $48.5 \%$ were $>7 \mathrm{~cm}$ in diameter, $60.6 \%$ were poorly differentiated, $52.3 \%$ were mucinous or medullary adenocarcinomas, $93.2 \%$ had an expanding pattern of growth, $40.9 \%$ showed intense peritumoral Crohn's-like lymphoid reaction, and only $21.2 \%$ were p53 positive by immunohistochemistry. A novel and interesting finding of our investigation was that the immunohistochemical pattern of MLH1/MSH2 expression is significantly related to several pathological features in MSI-H colorectal cancers. In particular, MLH1/MSH2positive carcinomas demonstrated pathological characteristics substantially different from those of MLH1-negative and MSH2-negative tumors and quite similar to those of MSI-L/MSS carcinomas. MSI-H carcinomas with positive MLH1 and MSH2 immunostaining were evenly distributed in the proximal and distal colon and less frequently displayed large size, poor differentiation, and mucinous or medullary histology than did MLH1negative and MSH2-negative tumors. Moreover, they often showed p53 protein overexpression (50\% of cases). Conversely, only minor pathological differences were observed between MLH1-negative and MSH2-negative carcinomas. Clinically, MSI-H MLH1/MSH2-positive carcinomas occurred more often among patients with HNPCC or suspected HNPCC and in the group of patients with one firstdegree relative affected by colorectal cancer.

All the MSI-H MLH1/MSH2-positive carcinomas demonstrated very high levels of MSI as well as unequivocal and diffuse nuclear staining for the two MMR proteins. In addition, all these tumors were classified as MSI-H when analyzed with the Bethesda consensus panel (data not shown), and all but one showed instability at both mononucleotide and dinucleotide repeats. In MSI-H MLH1/MSH2positive carcinomas, we cannot exclude the presence of MLH1 or MSH2 gene mutations not associated with loss of protein expression (36). However, our data suggest that probably other genes of the DNA mismatch repair complex or related genes play a major role in the development of these tumors.

Several studies demonstrated a better prognosis for patients with colorectal MSI-H carcinomas with respect to patients with MSI-L/MSS tumors (5, 1012). However, little is known about factors affecting the likelihood of survival among patients with MSI-H large bowel carcinomas (37-39). In the present investigation, we evaluated the prognostic significance of MLH1/MSH2 expression in 79 patients with MSI-H colorectal tumors and long-term follow-up. Patients with MLH1-negative carcinomas more frequently died of disease than did pa- 
tients with MSH2-negative and MLH1/MSH2positive carcinomas. Nevertheless, disease-specific survival analysis did not display significant differences between the three groups of patients. This may reflect the small numbers of cases in some of the groups being compared. Further studies are necessary to determine whether immunohistochemical pattern of MLH1/MSH2 expression influence survival in patients with MSI-H colorectal tumors.

According to the guidelines of the International Workshop of Bethesda (32), colorectal tumors with low levels of MSI are classified as MSI-L. The genetic bases of the MSI-L phenotype are not clearly defined, and the clinicopathological features of MSI-L large bowel carcinomas are not different from those of MSS tumors $(4,26)$. All the MSI-L cancers examined here demonstrated nuclear immunoreactivity for both MLH1 and MSH2 proteins. Our data confirm that MLH1 and MSH2 genes do not seem to be implicated in the etiology of MSI-L colorectal adenocarcinomas $(15,22,23)$.

Overall, the results of the present investigation strongly indicate that immunohistochemical analysis of MLH1 and MSH2 expression is a practical and reliable method for the detection of MSI-H large bowel adenocarcinomas. Our data also point out that MSI-H MLH1/MSH2-positive colorectal tumors are characterized by distinctive pathological features.

Acknowledgments: We thank Mr. Eros Mağri, Ms. Fernanda Mora, and Ms. Daniela Nardo for excellent technical assistance.

\section{REFERENCES}

1. Kinzler KW, Vogelstein B. Lessons from hereditary colorectal cancer. Cell 1996;87:159-70.

2. Toft NJ, Arends MJ. DNA mismatch repair and colorectal cancer. J Pathol 1998;185:123-9.

3. Kim H, Jen J, Vogelstein B, Hamilton SR. Clinical and pathological characteristics of sporadic colorectal carcinomas with DNA replication errors in microsatellite sequences. Am J Pathol 1994;145:148-56.

4. Jass JR, Do K-A, Simms LA, Iino H, Wynter C, Pillay SP, et al. Morphology of sporadic colorectal cancer with DNA replication errors. Gut 1998;42:673-9.

5. Gafà R, Maestri I, Matteuzzi M, Santini A, Ferretti S, Cavazzini L, et al. Sporadic colorectal adenocarcinoma with high-frequency microsatellite instability. Pathobiologic features, hMLH1 and hMSH2 expression, and clinical outcome. Cancer 2000;89:2025-37.

6. Rodriguez-Bigas MA, Boland CR, Hamilton SR, Henson DE, Jass JR, Khan PM, et al. A National Cancer Institute workshop on hereditary nonpolyposis colorectal cancer syndrome: meeting highlights and Bethesda guidelines. J Natl Cancer Inst 1997;89:1758-62.

7. Salovaara R, Loukola A, Kristo $P$, Kääriäinen $H$, Ahtola $H$, Eskelinen M, et al. Population-based molecular detection of hereditary nonpolyposis colorectal cancer. J Clin Oncol 2000; 18:2193-200.

8. Terdiman JP, Gum JR, Conrad PG, Miller GA, Weinberg V, Crawley SC, et al. Efficient detection of hereditary nonpolyposis colorectal cancer gene carriers by screening for tumor microsatellite instability before germline genetic testing. Gastroenterology 2001;120:21-30.

9. Thibodeau SN, Bren G, Schaid D. Microsatellite instability in cancer of the proximal colon. Science 1993;260:816-9.

10. Halling KC, French AJ, McDonnell SK, Burgart LJ, Schaid DJ, Peterson BJ, et al. Microsatellite instability and 8p allelic imbalance in stage B2 and C colorectal cancers. J Natl Cancer Inst 1999;91:1295-303.

11. Gryfe R, Kim H, Hsieh ETK, Aronson MD, Holowaty EJ, Bull $\mathrm{SB}$, et al. Tumor microsatellite instability and clinical outcome in young patients with colorectal cancer. N Engl J Med 2000;342:69-77.

12. Wright CM, Dent OF, Barker M, Newland RC, Chapuis PH, Bokey EL, et al. Prognostic significance of extensive microsatellite instability in sporadic clinicopathological stage C colorectal cancer. Br J Surg 2000;87:1197-202.

13. Elsaleh H, Joseph D, Grieu F, Zeps N, Spry N, Iacopetta B. Association of tumour site and sex with survival benefit from adjuvant chemotherapy in colorectal cancer. Lancet 2000; 355:1745-50.

14. Hemminki A, Mecklin J-P, Järvinen H, Aaltonen LA, Joensuu H. Microsatellite instability is a favorable prognostic indicator in patients with colorectal cancer receiving chemotherapy. Gastroenterology 2000;119:921-8.

15. Cawkwell L, Gray S, Murgatroyd H, Sutherland F, Haine L, Longfellow $\mathrm{M}$, et al. Choice of management strategy for colorectal cancer based on a diagnostic immunohistochemical test for defective mismatch repair. Gut 1999;45:409-15.

16. Wheeler JMD, Bodmer WF, Mortensen NJMcC. DNA mismatch repair genes and colorectal cancer. Gut 2000;47:14853.

17. Peltomäki P. Deficient DNA mismatch repair: a common etiologic factor for colon cancer. Hum Mol Genet 2001;10: $735-40$.

18. Cunningham JM, Christensen ER, Tester DJ, Kim C-Y, Roche PC, Burgart LJ, et al. Hypermethylation of the hMLH1 promoter in colon cancer with microsatellite instability. Cancer Res 1998;58:3455-60.

19. Herman JG, Umar A, Polyak K, Graff JR, Ahuja N, Issa J-PJ, et al. Incidence and functional consequences of MLH1 promoter hypermethylation in colorectal carcinoma. Proc Natl Acad Sci U S A 1998;95:6870-5.

20. Kuismanen SA, Holmberg MT, Salovaara R, de la Chapelle A, Peltomäki P. Genetic and epigenetic modification of MLH1 accounts for a major share of microsatellite-unstable colorectal cancers. Am J Pathol 2000;156:1773-9.

21. Thibodeau SN, French AJ, Roche PC, Cunningham JM, Tester DJ, Lindor NM, et al. Altered expression of hMSH2 and hMLH1 in tumors with microsatellite instability and genetic alterations in mismatch repair genes. Cancer Res 1996;56:4836-40.

22. Dietmaier W, Wallinger S, Bocker T, Kullmann F, Fishel R, Rüschoff J. Diagnostic microsatellite instability: definition and correlation with mismatch repair protein expression. Cancer Res 1997;57:4749-56.

23. Thibodeau SN, French AJ, Cunningham JM, Tester D, Burgart LJ, Roche PC, et al. Microsatellite instability in colorectal cancer: different mutator phenotypes and the principal involvement of hMLH1. Cancer Res 1998;58:1713-8.

24. Marcus VA, Madlensky L, Gryfe R, Kim H, So K, Millar A, et al. Immunohistochemistry for MLH1 and MSH2: a practical test for DNA mismatch repair-deficient tumors. Am J Surg Pathol 1999;23:1248-55. 
25. Chaves P, Cruz C, Lage P, Claro I, Cravo M, Leitão CN, et al. Immunohistochemical detection of mismatch repair gene proteins as a useful tool for the identification of colorectal carcinoma with the mutator phenotype. J Pathol 2000;191: 355-60.

26. Ward R, Meagher A, Tomlinson I, O'Connor T, Norrie M, Wu $\mathrm{R}$, et al. Microsatellite instability and the clinicopathological features of sporadic colorectal cancer. Gut 2001;48:821-9.

27. Sobin LH, Wittekind C, editors. UICC TNM classification of malignant tumours. 5th ed. New York: Wiley; 1997.

28. Jass JR, Sobin LH, editors. World Health Organization international histological classification of tumours. Histological typing of intestinal tumours. 2nd ed. Berlin: Springer-Verlag; 1989.

29. Lanza G, Gafà R, Matteuzzi M, Santini A. Medullary-type poorly differentiated adenocarcinoma of the large bowel: a distinct clinicopathologic entity characterized by microsatellite instability and improved survival. J Clin Oncol 1999;17: 2429-38.

30. Jass JR, Atkin WS, Cuzick J, Bussey HJR, Morson BC, Northover JMA, et al. The grading of rectal cancer: historical perspectives and a multivariate analysis of 447 cases. Histopathology 1986;10:437-59.

31. Graham DM, Appelman HD. Crohn's-like lymphoid reaction and colorectal carcinoma: a potential histologic prognosticator. Mod Pathol 1990;3:332-5.

32. Boland CR, Thibodeau SN, Hamilton SR, Sidransky D, Eshleman JR, Burt RW, et al. A National Cancer Institute workshop on microsatellite instability for cancer detection and familial predisposition: development of international criteria for the determination of microsatellite instability in colorectal cancer. Cancer Res 1998;58:5248-57.
33. Bocker Edmonston T, Cuesta KH, Burkholder S, Barusevicius A, Rose D, Kovatich AJ, et al. Colorectal carcinomas with high microsatellite instability: defining a distinct immunologic and molecular entity with respect to prognostic markers. Hum Pathol 2000;31:1506-14.

34. Dolcetti R, Viel A, Doglioni C, Russo A, Guidoboni M, Capozzi E, et al. High prevalence of activated intraepithelial cytotoxic $\mathrm{T}$ lymphocytes and increased neoplastic cell apoptosis in colorectal carcinomas with microsatellite instability. Am J Pathol 1999;154:1805-13.

35. Samowitz WS, Holden JA, Curtin K, Edwards SL, Walker AR, Lin HA, et al. Inverse relationship between microsatellite instability and K-ras and p53 gene alterations in colon cancer. Am J Pathol 2001;158:1517-24.

36. Salahshor S, Koelble K, Rubio C, Lindblom A. Microsatellite instability and hMLH1 and hMSH2 expression analysis in familial and sporadic colorectal cancer. Lab Invest 2001;81: 535-41.

37. Guidoboni M, Gafà R, Viel A, Doglioni C, Russo A, Santini A, et al. Microsatellite instability and high content of activated cytotoxic lymphocytes identify colon cancer patients with a favorable prognosis. Am J Pathol 2001;159: 297-304.

38. Watanabe T, Wu T-T, Catalano PJ, Ueki T, Satriano R, Haller DG, et al. Molecular predictors of survival after adjuvant chemotherapy for colon cancer. N Engl J Med 2001;344: 1196-206.

39. Yamamoto H, Yamashita K, Perucho M. Somatic mutation of the $\beta 2$-microglobulin gene associates with unfavorable prognosis in gastrointestinal cancer of the microsatellite mutator phenotype [letter]. Gastroenterology 2001;120: 1565-7.
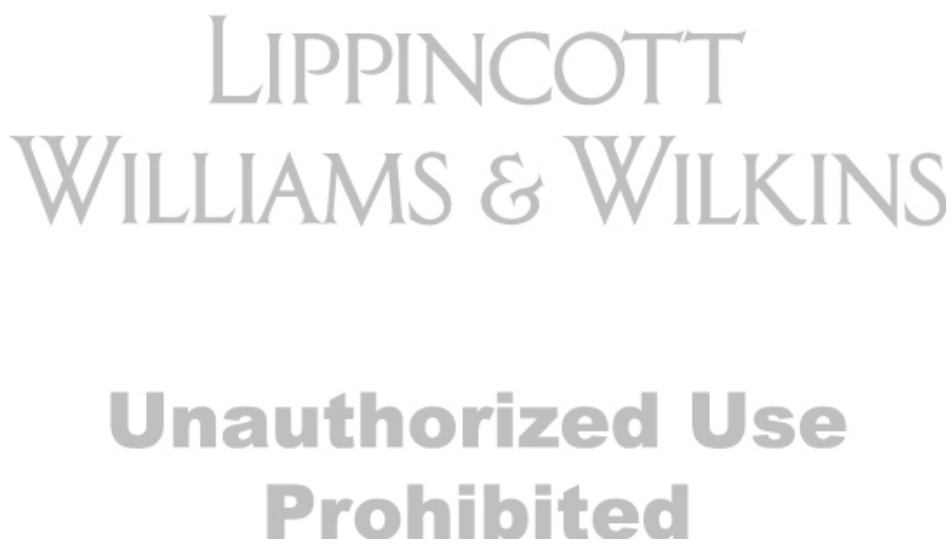